\title{
Efflux pumps expression and its association with porin down-regulation and $\beta$-lactamase production among Pseudomonas aeruginosa causing bloodstream infections in Brazil
}

\author{
Danilo E Xavier*, Renata C Picão, Raquel Girardello, Lorena CC Fehlberg, Ana C Gales
}

\begin{abstract}
Background: Multi-drug efflux pumps have been increasingly recognized as a major component of resistance in $P$. aeruginosa. We have investigated the expression level of efflux systems among clinical isolates of $P$. aeruginosa, regardless of their antimicrobial susceptibility profile.

Results: Aztreonam exhibited the highest in vitro activity against the $P$. aeruginosa isolates studied $(64.4 \%$ susceptibility), whereas susceptibility rates of imipenem and meropenem were both $47.5 \%$. The MexXY-OprM and MexAB-OprM efflux systems were overexpressed in $50.8 \%$ and $27.1 \%$ of isolates studied, respectively. Overexpression of the MexEF-OprN and MexCD-OprJ systems was not observed. AmpC $\beta$-lactamase was overexpressed in $11.9 \%$ of $P$. aeruginosa isolates. In addition, decreased oprD expression was also observed in $69.5 \%$ of the whole collection, and in $87.1 \%$ of the imipenem non-susceptible P. aeruginosa clinical isolates. The MBLencoding genes bla SPM-1 $_{1}$ and bla $a_{\mathrm{IMP}-1}$ were detected in $23.7 \%$ and $1.7 \% \mathrm{P}$. aeruginosa isolates, respectively. The bla $a_{\mathrm{GES}-1}$ was detected in $5.1 \%$ of the isolates, while bla $a_{\mathrm{GES}-5}$ and bla $a_{\mathrm{CTX}-\mathrm{M}-2}$ were observed in $1.7 \%$ of the isolates evaluated. In the present study, we have observed that efflux systems represent an adjuvant mechanism for antimicrobial resistance.
\end{abstract}

Conclusions: Efflux systems in association of distinct mechanisms such as the porin down-regulation, AmpC overproduction and secondary $\beta$-lactamases play also an important role in the multi-drug resistance phenotype among $P$. aeruginosa clinical isolates.

\section{Background}

Pseudomonas aeruginosa is an aerobic gram-negative pathogen and a common etiologic agent of nosocomial infections, especially pneumonia, in seriously ill patients $[1,2]$. This species is intrinsically resistant to many antimicrobial agents and usually develop resistance to other antimicrobial agents during antimicrobial chemotherapy, further limiting the available therapeutic options [3].

Bacterial efflux systems capable of ejecting antimicrobials are mostly encoded by chromosomal genes and generally fall into five classes, the major facilitator superfamily (MFS), the ATP-binding cassette (ABC)

\footnotetext{
*Correspondence: danilo.elias@gmail.com

Division of Infectious Diseases, Universidade Federal de São Paulo, Rua Pedro de Toledo, 781, 04039-032, São Paulo, Brazil
}

family, the small multi-drug resistance (SMR) family, the multi-drug and toxic compound extrusion (MATE) family and the resistance-nodulation-division (RND) family [4]. The RND chromosomal systems are encoded by operons and are typically formed by three proteins, which are located in the inner membrane, periplasm and outer membrane of the bacterial cell [5].

Sequencing of $P$. aeruginosa genome revealed the presence of several RND efflux systems. Of those, MexABOprM, MexCD-OprJ, MexEF-OprN and MexXY-OprM are able to pump out multiple antipseudomonal compounds $[1,4,6]$. Studies with MexAB-OprM mutants demonstrated that this efflux system extrudes quinolones, aminoglycosides, macrolides, tetracycline, chloramphenicol, novobiocin, and most $\beta$-lactams but not imipenem [5]. The MexXY-OprM is able to eject 
cefepime, cefotaxime, levofloxacin, ciprofloxacin, amikacin, gentamicin, tobramycin, erythromycin, tetracycline and meropenem [5]. MexAB-OprM and MexXY-OprM are constitutively expressed and contribute to the intrinsic resistance phenotype of $P$. aeruginosa. However, when overexpressed, these efflux systems confer reduced susceptibility to different classes of antimicrobial agents $[7,8]$. Although the efflux systems MexCD-OprJ and MexEF-OprN are quiescent in wild type $P$. aeruginosa, their overexpression may also contribute to the acquired multi-drug resistance phenotype in mutant isolates [5].

Overexpression of efflux systems generally confers modest levels of antimicrobial resistance $[9,10]$. However, its association with other resistance determinants is frequently observed [11]. In Brazil, production of extended-spectrum $\beta$-lactamases (ESBL), such as CTX-M (cefotaximase) and GES (Guiana-extended spectrum), or metallo- $\beta$-lactamases (MBL) such as SPM (São Paulo Metallo- $\beta$-lactamase) and IMP (imipenemase) are the main mechanisms of acquired resistance to broadspectrum $\beta$-lactams among $P$. aeruginosa clinical isolates [12]. The association of these $\beta$-lactamases with overexpression of efflux pumps and/or porin loss may lead to high level and/or co-resistance phenotypes [11]. For this reason, efflux pumps may seriously impact antimicrobial therapy in clinical settings. The aim of this study was to investigate the expression of efflux systems as well as its association with other resistance mechanisms, such as $\beta$-lactamase production and porin down-regulation, among $P$. aeruginosa clinical isolates.

\section{Results}

Bacterial isolates and antimicrobial susceptibility profile

Fifty-nine non-repetitive $P$. aeruginosa isolates were collected from bloodstream infections between June and December 2005. The majority of isolates was collected from patients hospitalized in intensive care units (64.4\%), followed by the emergency room ward (28.8\%) and pediatric oncology unit (6.8\%). Aztreonam showed the highest susceptibility rate against the isolates studied (64.4\%), followed by cefepime $(49.2 \%)$, meropenem (47.2\%), imipenem (47.2\%), ceftazidime (44.1\%), amikacin $(40.7 \%)$, ciprofloxacin $(35.6 \%)$ and gentamicin (32.2\%, Table 1). Approximately $17 \%$ of the isolates $(n=10)$ were susceptible to all tested antimicrobial.

\section{Pulsed Field Gel Electrophoresis}

A total of 23 distinct PFGE patterns were detected among the $59 \mathrm{P}$. aeruginosa clinical isolates studied. Five $P$. aeruginosa isolates could not be typed by PFGE using SpeI. Although 38 isolates were clustered in six PFGE patterns, 16 isolates showed distinct PFGE patterns.

\section{Carbapenems hydrolysis and $\boldsymbol{\beta}$-lactamases production}

Carbapenem hydrolysis was detected in $15 \mathrm{P}$. aeruginosa, representing $25.4 \%$ of the whole collection and $48.4 \%$ of the imipenem-resistant isolates. These isolates had their carbapenemase activity inhibited by EDTA, and the presence of the MBL-encoding genes $b l a_{\mathrm{SPM}-1}$ and $b l a_{\mathrm{IMP}}$-like was confirmed by multiplex $\mathrm{PCR}$, in 14 and 1 isolates, respectively. Among the SPM-producing $P$. aeruginosa studied, 13 showed the same PFGE pattern, whereas one isolate could not be typed using Spe I. ESBL-encoding genes were present in five isolates: $b l a_{\mathrm{GES}-1}(n=3), \operatorname{bla}_{\mathrm{GES}-5}(n=1)$ and bla $a_{\mathrm{CTX}-\mathrm{M}-2}(n=1)$. GES-type producers belonged to the same genotype, whereas CTX-M-2-producer showed a unique PFGE profile.

\section{Gene expression}

The percentage of $P$. aeruginosa isolates that were nonsusceptible to antimicrobials and demonstrated overexpression of efflux genes and $a m p C$, coupled with oprD down-regulation is shown in Table 1 . In addition, Table 2 shows the association of different resistance mechanisms identified, and antimicrobials MICs that were more frequently observed at each association (modal MIC).

The gene expression analysis showed that $50.8 \%$ $(n=30)$ and $27.1 \%(n=16)$ of $P$. aeruginosa clinical isolates demonstrated increased mex $Y$ (from 2.2- to 41.0fold) and mexB (from 2.1 - to 10.0-fold) transcription mRNA levels, respectively, compared to those of PAO1. In addition, $11 P$. aeruginosa isolates (18.6\%) showed overexpression of both mexB and mex $Y$ efflux genes. Overexpression of MexCD-OprJ and MexEF-OprN were not observed among the clinical isolates of $P$. aeruginosa evaluated in this study. Overall, $69.5 \%$ and $11.9 \%$ of $P$. aeruginosa clinical isolates studied showed decreased oprD expression (from 0.1 - to 0.7-fold compared to PAO1), and overexpression of $a m p C$ (from 14- to 402fold compared to PAO1), respectively. None of the investigated resistance determinants was identified in $11.8 \%$ of clinical isolates $(n=7$, Table 2$)$.

Among the isolates overexpressing the mex $Y$ efflux gene, $86.7 \%$ were not susceptible to amikacin, gentamicin and ciprofloxacin. Cefepime non-susceptibility was observed in $80 \%$ of isolates overexpressing mexY. Of those, $79.2 \%$ also presented reduced oprD transcription, $54.2 \%$ were MBL-producers, $12.5 \%$ produced the ESBL GES-1, and $16.7 \%$ showed increased ampC transcriptional levels (data not shown). Among the cefepime non-susceptible isolates that did not show mex $Y$ overexpression, $33.3 \%$ produced SPM-1, 33.3\% overexpressed $a m p C, 16.7 \%$ produced the ESBL CTX-M-2, and $16.7 \%$ produced GES-5, an ESBL with carbapenemase activity. 
Table 1 The percentage of $P$. aeruginosa isolates that were non-susceptible to antimicrobials and demonstrated overexpression of efflux genes and $\operatorname{ampC} \beta$-lactamase, coupled with oprD down-regulation

\begin{tabular}{lccccc}
\hline Antimicrobial & Non-susceptible $(\boldsymbol{n}=\mathbf{5 9 )}$ & \multicolumn{3}{c}{ \% of isolates $(\boldsymbol{n})$} \\
\cline { 3 - 6 } & & $\mathbf{A B M + ( 1 6 )}$ & $\mathbf{X Y}+(\mathbf{3 0})$ & AmpC+ (07) & OprD- (41) \\
\hline Aztreonam & $21(35.6)$ & $56.3(09)$ & $43.3(13)$ & $71.4(05)$ & $34.1(14)$ \\
\hline Imipenem & $31(52.5)$ & $56.3(09)$ & $80.0(24)$ & $71.4(05)$ & $65.9(27)$ \\
\hline Meropenem & $31(52.5)$ & $62.5(10)$ & $80.0(24)$ & $71.4(05)$ & $63.4(26)$ \\
\hline Cefepime & $30(50.8)$ & $56.3(09)$ & $80.0(24)$ & $85.7(06)$ & $58.5(24)$ \\
\hline Ceftazidime & $33(55.9)$ & $50.0(08)$ & $76.7(23)$ & $100(07)$ & $63.4(26)$ \\
\hline Amikacin & $35(59.3)$ & $68.8(11)$ & $86.7(26)$ & $57.1(04)$ & $70.7(29)$ \\
\hline Gentamicin & $40(67.8)$ & $75.0(12)$ & $86.7(26)$ & $57.1(04)$ & $65.9(27)$ \\
\hline Ciprofloxacin & $38(64.4)$ & $81.3(13)$ & $86.7(26)$ & $85.7(06)$ & $63.4(26)$ \\
\hline
\end{tabular}

The abbreviations $\mathrm{ABM}+, \mathrm{XY}+$ and $\mathrm{AmpC}+$ designate MexAB-OprM, MexXY, and AmpC overexpression, respectively.

OprD -: OprD porin down-regulation.

Meropenem non-susceptibility was observed among $62.5 \%$ of isolates overexpressing $\operatorname{mexB}$ (from 2.1- to 5.5fold higher than PAO1). Of those, $90.0 \%$ showed decreased oprD expression, $40.0 \%$ were MBL producers, $20.0 \%$ overexpressed $a m p C$ and $10.0 \%$ were GES-5 producers (data not shown). As expected, all meropenemsusceptible isolates that overexpressed $\operatorname{mexB}$, presented normal expression of both $\operatorname{amp} C$ and $\operatorname{opr} D$ when compared to that of PAO1. Higher percentage of $\operatorname{mex} B$ overexpression was observed among isolates that were also not susceptible to cefepime, amikacin, gentamicin and ciprofloxacin. Of note, $85.7 \%$ and $28.6 \%$ of SPMproducing $P$. aeruginosa showed increased transcriptional levels of mex $Y$ and mexB, respectively.

It is worth to mention that MexAB-OprM and/or MexXY-OprM overexpression was observed among isolates that were susceptible to most antimicrobials tested. This finding was expected since efflux pump

Table 2 Association of resistance mechanisms identified among the $P$. aeruginosa isolates $(n=59)$ and the modal MICs for tested antimicrobials observed in each association

\begin{tabular}{|c|c|c|c|c|c|c|c|c|c|}
\hline \multicolumn{2}{|c|}{$\begin{array}{l}\text { Isolates and determinant of antimicrobial resistance (No. of } \\
\text { isolates with OprD -) }\end{array}$} & \multicolumn{8}{|c|}{ Modal MIC ${ }^{\mathrm{a}}(\mu \mathrm{g} / \mathrm{ml})$} \\
\hline & & \multirow{2}{*}{$\begin{array}{c}\text { MER } \\
0.25\end{array}$} & \multirow{2}{*}{$\frac{\text { IPM }}{1}$} & \multirow{2}{*}{$\frac{\text { ATM }}{4}$} & \multirow{2}{*}{$\frac{\text { CAZ }}{0.5}$} & \multirow{2}{*}{$\begin{array}{l}\text { FEP } \\
0.25\end{array}$} & \multirow{2}{*}{$\begin{array}{c}\text { AMK } \\
2\end{array}$} & \multirow{2}{*}{$\frac{\text { GEN }}{4}$} & \multirow{2}{*}{$\frac{\text { CIP }}{0.25-}$} \\
\hline PAO1 reference strain & - & & & & & & & & \\
\hline No mechanisms of resistance identified & $7(0)$ & 4 & 2 & $4-8^{b}$ & 4 & 2 & 8 & 4 & 16 \\
\hline$X Y+, M B L$ & $7(6)$ & $>32$ & $>32$ & 8 & 256 & $>32$ & $>256$ & $>256$ & $>32$ \\
\hline$X Y+$ & $7(5)$ & 16 & $8 / 16^{b}$ & 32 & $8 / 256^{b}$ & $>32$ & 256 & $2 />256^{b}$ & $0.5 />32^{b}$ \\
\hline $\mathrm{ABM}+, \mathrm{XY}+$ & $5(2)$ & $0.25 / 8^{b}$ & $0.25 / 2^{b}$ & 16 & 8 & 4 & 256 & $2->256^{c}$ & 32 \\
\hline $\mathrm{ABM}+, \mathrm{XY}+, \mathrm{MBL}$ & $4(3)$ & $>32$ & $>32$ & 8 & 256 & $>32$ & $>256$ & $>256$ & $>32$ \\
\hline $\mathrm{ABM+}$ & $3(2)$ & $0.5-16^{\mathrm{b}}$ & 1 & 16 & $2-8^{c}$ & 4 & $4-32^{c}$ & $1-8^{c}$ & $0.25-8^{c}$ \\
\hline$X Y+, G E S-1$ & $3(2)$ & $8->32^{c}$ & $8^{-}>32^{c}$ & 8 & 128 & $>32$ & $>256$ & 256 & 16 \\
\hline $\mathrm{ABM}+, \mathrm{XY}+, \mathrm{AmpC}+$ & $2(2)$ & $16 />32^{b}$ & $>32$ & $8 / 32^{b}$ & $32 / 64^{b}$ & $16 / 32^{b}$ & $4 / 64^{b}$ & $1 / 8^{b}$ & $2 / 4^{b}$ \\
\hline $\mathrm{ABM}+, \mathrm{GES}-5$ & $1(1)$ & $>32$ & 32 & 8 & 32 & $>32$ & 128 & 128 & 32 \\
\hline $\mathrm{ABM}+, \mathrm{CTX}-\mathrm{M} 2$ & $1(1)$ & 4 & 1 & $>32$ & 2 & $>32$ & 128 & 256 & 16 \\
\hline $\mathrm{XY}+, \mathrm{AmpC}+, \mathrm{MBL}$ & $2(2)$ & $32 />32^{b}$ & $>32$ & $16 />32^{b}$ & $128 />256^{b}$ & $>32$ & $>256$ & $>256$ & $>32$ \\
\hline MBL & $2(2)$ & $>32$ & $>32$ & 8 & 256 & $>32$ & $>256$ & $>256$ & 32 \\
\hline $\mathrm{AmpC}+$ & $3(2)$ & $1-8$ & $2-16^{c}$ & $4-32^{c}$ & $16-256^{c}$ & 16 & 4 & 2 & $0.5-32^{c}$ \\
\hline OprD- & $12(12)$ & $\leq 0.25$ & $1-2$ & 8 & 2 & 2 & 8 & 2 & 0.25 \\
\hline
\end{tabular}

MER, meropenem; IPM, imipenem; ATM, aztreonam; CAZ, ceftazidime; FEP, cefepime; AMK, amikacin; GEN, gentamicin; CIP, ciprofloxacin.

The abbreviations $\mathrm{XY}+\mathrm{ABM}+$, and $\mathrm{AmpC}+$ designate MexXY, MexAB-OprM, and AmpC overexpression, respectively.

$\mathrm{MBL}$, metallo- $\beta$-lactamase producer

OprD-, reduced expression of OprD porin.

a, Modal MIC is defined as the antimicrobial MICs that were more frequently observed at each association of resistance mechanisms.

b, two modal MICs observed;

${ }^{c}$ MIC range when no modal MIC was observed. 
overexpression in $P$. aeruginosa usually confers modest increase in the MICs of antimicrobial agents that are ejected by these systems.

\section{Discussion and Conclusions}

$P$. aeruginosa is the fifth most frequent pathogen of bloodstream infections and the first one causing pneumonia in Latin America according to the SENTRY Antimicrobial Surveillance Program [13]. In the last decades, the emergency of multi-drug resistant $P$. aeruginosa has been observed worldwide. Some of antimicrobial agents have become less effective against these organisms reducing the available therapeutic options for treatment of these infections.

In this study $52.5 \%$ of the $P$. aeruginosa isolates studied were resistant to carbapenems. Our findings are in accordance of previous studies that showed high rates of antimicrobial resistance, including carbapenems, among $P$. aeruginosa clinical isolates collected from Brazilian institutions [14]. The genetic diversity observed among the $P$. aeruginosa isolates studied indicates that spread of clones and emergency of distinct genotypes have occurred in our hospital. The high rate of carbapenem resistance can be partially explained by the spread of an endemic SPM-producing clone. It also justifies the susceptibility rate to aztreonam since MBL producers are not able to hydrolyze this antimicrobial agent. This finding corroborates with those previously reported that described a single SPM producer clone spread out in the Brazilian territory [15].

The overexpression of efflux systems may impact on clinical outcome of $P$. aeruginosa infections since they are capable of pumping out many classes of antimicrobial agents used for treatment of these infections [16]. However, it has not been clearly established the correlation between increase in the transcriptional level of an efflux-encoding gene and antimicrobial resistance leading to possible therapeutic failure [17].

In the present study, we have evaluated the transcriptional levels of four efflux-encoding genes as well as ampC and oprD among $59 \mathrm{P}$. aeruginosa clinical isolates. This collection represents the total number of patients with bloodstream infection due to $P$. aeruginosa in a six-month period in Hospital São Paulo, Brazil. We also aimed to evaluate the frequency of isolates presenting different mechanisms of $\beta$-lactam resistance and their association.

The overexpression of the MexAB-OprM and MexXY-OprM efflux systems were more frequent among antimicrobial resistant $P$. aeruginosa isolates. Since MexAB-OprM and MexXY-OprM are constitutively expressed in wild type $P$. aeruginosa isolates, the antimicrobial policy in use in each individual institution may interfere with the selection of the most overexpressed efflux system. Aminoglycosides are important substrates of MexXY-OprM and might have exerted a role in selecting $P$. aeruginosa that overexpressed this system [18]. The expression of MexXYOprM is inducible, while expression of MexAB-OprM is not [5]. In our institution, the prescription of aminoglycosides is not controlled and these antimicrobial agents usually are prescribed in combination for treatment of $P$. aeruginosa infections. These facts could in part justify why MexXY-OprM was the most frequent overexpressed efflux system, since mex $X Y$ expression may be induced by these antimicrobial class [19]. Interestingly, the overexpression of MexXY-OprM was observed in all MBL-producing isolates.

We did not notice a strict correlation between antimicrobial resistance and efflux genes overexpression. However, efflux overexpressing isolates often presented higher antimicrobial MICs than did PAO1 and those isolates in which no antimicrobial resistance determinant was found.

Our findings clearly demonstrate that $\beta$-lactamase production increase antimicrobial MICs more efficiently than do efflux overexpression or porin down-regulation alone. However, these chromosomal resistance mechanisms were frequently present among acquired $\beta$-lactamase producers. These findings suggest that efflux overexpression and porin down-regulation may favor the bacterial survival under selective pressure, increasing its chance to acquire further resistance determinants.

In the present study we have observed that efflux pump overexpression do not appear to be the main mechanism of drug resistance among the studied clinical isolates of $P$. aeruginosa, but represents an adjuvant mechanism for antimicrobial resistance. The association of distinct mechanisms such as the porin down-regulation and AmpC overproduction play also an important role in the multi-drug resistance phenotype among $P$. aeruginosa clinical isolates studied. In addition, our findings indicate that spread of clones and emergency of distinct genotypes have occurred in our institution and implementation of control measures is extremely necessary to modify this scenario.

\section{Methods}

Bacterial isolates and antimicrobial susceptibility testing With the approval of the local Ethics in Research committee (Comitê de Ética em Pesquisa Hospital São Paulo, protocol number: CEP0398/07), a total of 59 clinical isolates of $P$. aeruginosa were evaluated, regardless of their antimicrobial susceptibility profile. These isolates were consecutively collected between June and December 2005 from blood culture of patients hospitalized at Hospital São Paulo, a tertiary teaching hospital located in São Paulo, Brazil. Only a single bacterial 
isolate per patient was evaluated. MICs for ceftazidime, cefepime, aztreonam, imipenem, meropenem, gentamicin, amikacin and ciprofloxacin were determined by agar dilution and interpreted according to Clinical Laboratory Standards Institute [20,21]. P. aeruginosa ATCC 27853 and Escherichia coli ATCC 25922 strains were used as quality control strains.

\section{Pulsed Field Gel Electrophoresis}

Genomic DNA of isolates was prepared in agarose blocks and digested with the restriction enzyme SpeI (New England, Beverly, MA). Electrophoresis was performed on CHEF-DR III (BioRad, Richmond, CA), with the following conditions: $0.5 \times \mathrm{TBE}, 1 \%$ agarose, $13^{\circ} \mathrm{C}$, $200 \mathrm{~V}$, for $24 \mathrm{~h}$ with switch time ramped from 5 to $90 \mathrm{~s}$. The band patterns were interpreted as previously recommended [22].

\section{Screening for carbapenemase producers and detection of} $\beta$-lactamases-encoding genes

Investigation of carbapenemase activity in crude extracts was performed by UV spectrophotometric assays. Briefly, a full $10 \mu \mathrm{l}$ loop of the test organism was inoculated into $500 \mu \mathrm{l}$ of phosphate buffer $100 \mathrm{mM}(\mathrm{pH} \mathrm{7.0)}$ and disrupted by sonication. The cells were removed by centrifugation and the supernatants were used for further experiments. Protein quantification in the crude extracts was performed using the Bradford stain. Hydrolytic activity of crude extracts was determined against $100 \mu \mathrm{M}$ imipenem and $100 \mu \mathrm{M}$ meropenem in $100 \mathrm{mM}$ phosphate buffer ( $\mathrm{pH}$ 7.0). Measurements were carried out at a $297 \mathrm{~nm}$ wavelength. Positive control included SPM-1producing $P$. aeruginosa 48-1997A [23]. Carbapenem hydrolysis inhibition was performed by incubating the crude extract with $25 \mathrm{mM}$ EDTA during $15 \mathrm{~min}$, previously to the assay with imipenem and meropenem. Detection MBL-encoding genes was performed for all carbapenem-resistant isolates by multiplex PCR, as previously described [24]. The presence of ESBLencoding genes bla $a_{\mathrm{TEM}}, b l a_{\mathrm{SHV}}, b l a_{\mathrm{CTX}-\mathrm{M}}, b l a_{\mathrm{GES}}, b l a_{\mathrm{VEB}}$ and $b l a_{\mathrm{PER}}$ was investigated by PCR, as previously reported [12,25].

\section{Quantitative RT-PCR (RT-qPCR)}

Transcriptional levels of $\operatorname{mex} B, \operatorname{mex} D, \operatorname{mex} F, \operatorname{mex} Y$, $a m p C$ and $o p r D$ were determined with Mastercycler Realplex ${ }^{2}$ (Eppendorf, Hamburg, Germany). In brief, total RNA was extracted using the RNase Mini Kit, following the manufacturer recommendations (Qiagen, Hilden, Germany). Five micrograms of total RNA was submitted to cDNA synthesis using High Capacity cDNA Archive Kit (Applied Biosystems, Foster City, USA). Quantitative RT-PCR was performed with Platinum SYBR Green Supermix (Invitrogen, Carlsbad, USA), using specific primers for mex $B, \operatorname{mex} D, \operatorname{mex} F$, $m e x Y$, ampC and oprD as previously described [26-29] or designed for this study using the GeneFisher online software http://bibiserv.techfak.uni-bielefeld.de/genefisher/old.html (Table 3). Amplification was carried out in triplicate from cDNA preparations. To assure that specific amplification had occurred, melting curves of each amplicon was assessed and compared to that Tm obtained when using PAO1 DNA total was tested as template. A gene encoding the ribosomal protein $r p s L$ was used as a reference gene for normalizing the transcriptional levels of target genes. Transcription data were analyzed with the Q-Gene software [30]. According to previous studies [31] the efflux systems MexAB-

Table 3 Primers used in this study for access the relative gene expression by RT-qPCR

\begin{tabular}{|c|c|c|c|c|}
\hline Genes & Primers & Sequences $\left(5^{\prime}-3^{\prime}\right)$ & Amplicon size (bp) & References \\
\hline \multirow[t]{2}{*}{$\operatorname{mex} B$} & mexB-F & GTGTTCGGCTCGCAGTACTC & 244 & {$[26]$} \\
\hline & mexB-R & AACCGTCGGGATTGACCTTG & & \\
\hline \multirow[t]{2}{*}{$\operatorname{mexD}$} & mexD-F & CGAGCGCTATTCGCTGC & 165 & This study \\
\hline & mexD-R & GGCAGTTGCACGTCGA & & \\
\hline \multirow[t]{2}{*}{ mexF } & mexF-F & CGCCTGGTCACCGAGGAAGAGT & 255 & {$[27]$} \\
\hline & mexF-R & TAGTCCATGGCTTGCGGGAAGC & & \\
\hline \multirow[t]{2}{*}{ mexy } & mexY-F & CCGCTACAACGGCTATCCCT & 250 & {$[26]$} \\
\hline & mexY-R & AGCGGGATCGACCAGCTTC & & \\
\hline \multirow[t]{2}{*}{ oprD } & oprD-F & TCCGCAGGTAGCACTCAGTTC & 191 & {$[28]$} \\
\hline & oprD-R & AAGCCGGATTCATAGGTGGTG & & \\
\hline \multirow[t]{2}{*}{$a m p C$} & ampC-F & CTGTTCGAGATCGGCTC & 166 & This study \\
\hline & $\mathrm{ampC}-\mathrm{R}$ & CGGTATAGGTCGCGAG & & \\
\hline \multirow[t]{2}{*}{ rpsL } & rpsL-F & GCAAGCGCATGGTCGACAAGA & 201 & {$[29]$} \\
\hline & rpsL-R & CGCTGTGCTCTTGCAGGTTGTGA & & \\
\hline
\end{tabular}


OprM, MexCD-OprJ, MexEF-OprN, and MexXY were considered overexpressed when the transcriptional levels of $\operatorname{mex} B, \operatorname{mex} C, \operatorname{mex} E$, and $\operatorname{mex} Y$ were at least 2,100 , 100 , and 4 fold higher than those of the wild-type reference strain PAO1, respectively. Reduced oprD expression and overexpression of $a m p C$ were considered relevant when their transcriptional levels were $\leq 70 \%$ and $\geq 10$-fold, respectively, compared to that of the PAO1 reference strain $[10,32]$.

\section{Competing interests}

The authors declare that they have no competing interests.

\section{Authors' contributions}

All authors had equal contribution in preparing this article. DEX drafted the first manuscript of this article based on his MSc thesis, which was supervised by RCP and ACG. RG was involved in the determination of antimicrobial susceptible profile. LCCF carried out the molecular typing and was involved in the determination of the gene transcriptional level. All authors read and approved the final manuscript.

\section{Funding}

This work was financially supported by Fundação de Amparo à Pesquisa do Estado de São Paulo (FAPESP - 2006/01716-8), by Coordenação de Aperfeiçoamento de Pessoal de Nível Superior (CAPES) that conceded a grant to DEX and Conselho Nacional de Desenvolvimento Científico e Tecnológico (CNPq) that provides a researcher grant to ACG. (307714/20063).

\section{Acknowledgements}

We would like to thank Soraya S. Andrade for the critical reading of this manuscript.

Received: 1 March 2010 Accepted: 12 August 2010

Published: 12 August 2010

\section{References}

1. Stover CK, Pham XQ, Erwin AL, Mizoguchi SD, Warrener P, Hickey MJ, Brinkman FS, Hufnagle WO, Kowalik DJ, Lagrou M, et al: Complete genome sequence of Pseudomonas aeruginosa PAO1, an opportunistic pathogen. Nature 2000, 406:959-964.

2. Engel J, Balachandran P: Role of Pseudomonas aeruginosa type III effectors in disease. Curr Opin Microbiol 2009, 12:61-66.

3. Dotsch A, Becker T, Pommerenke C, Magnowska Z, Jansch L, Haussler S: Genomewide identification of genetic determinants of antimicrobial drug resistance in Pseudomonas aeruginosa. Antimicrob Agents Chemother 2009, 53:2522-2531.

4. Poole K: Efflux pumps as antimicrobial resistance mechanisms. Ann Med 2007, 39:162-176.

5. Poole K, Srikumar R: Multidrug efflux in Pseudomonas aeruginosa: components, mechanisms and clinical significance. Curr Top Med Chem 2001, 1:59-71

6. Poole K: Resistance to beta-lactam antibiotics. Cell Mol Life Sci 2004 61:2200-2223

7. Sobel ML, Hocquet D, Cao L, Plesiat P, Poole K: Mutations in PA3574 (nalD) lead to increased MexAB-OprM expression and multidrug resistance in laboratory and clinical isolates of Pseudomonas aeruginosa. Antimicrob Agents Chemother 2005, 49:1782-1786.

8. Cao L, Srikumar R, Poole K: MexAB-OprM hyperexpression in NalC-type multidrug-resistant Pseudomonas aeruginosa: identification and characterization of the nalC gene encoding a repressor of PA3720PA3719. Mol Microbiol 2004, 53:1423-1436.

9. Lee A, Mao W, Warren MS, Mistry A, Hoshino K, Okumura R, Ishida H, Lomovskaya O: Interplay between efflux pumps may provide either additive or multiplicative effects on drug resistance. J Bacteriol 2000, 182:3142-3150.
10. Quale J, Bratu S, Gupta J, Landman D: Interplay of efflux system, ampC, and oprD expression in carbapenem resistance of Pseudomonas aeruginosa clinical isolates. Antimicrob Agents Chemother 2006, 50:1633-1641.

11. Tomas M, Doumith M, Warner M, Turton JF, Beceiro A, Bou G, Livermore DM, Woodford N: Efflux pumps, OprD porin, AmpC betalactamase, and multiresistance in Pseudomonas aeruginosa isolates from cystic fibrosis patients. Antimicrob Agents Chemother 2010, 54:2219-2224.

12. Picao RC, Poirel L, Gales AC, Nordmann P: Diversity of beta-lactamases produced by ceftazidime-resistant Pseudomonas aeruginosa isolates causing bloodstream infections in Brazil. Antimicrob Agents Chemother 2009, 53:3908-3913.

13. Andrade SS, Jones RN, Gales AC, Sader HS: Increasing prevalence of antimicrobial resistance among Pseudomonas aeruginosa isolates in Latin American medical centres: 5 year report of the SENTRY Antimicrobial Surveillance Program (1997-2001). J Antimicrob Chemother 2003, 52:140-141.

14. Marra AR, Pereira CA, Gales AC, Menezes LC, Cal RG, de Souza JM, Edmond MB, Faro C, Wey SB: Bloodstream infections with metallo-betalactamase-producing Pseudomonas aeruginosa: epidemiology, microbiology, and clinical outcomes. Antimicrob Agents Chemother 2006, 50:388-390.

15. Gales AC, Menezes LC, Silbert S, Sader HS: Dissemination in distinct Brazilian regions of an epidemic carbapenem-resistant Pseudomonas aeruginosa producing SPM metallo-beta-lactamase. J Antimicrob Chemother 2003, 52:699-702.

16. Li XZ, Nikaido H: Efflux-mediated drug resistance in bacteria: an update. Drugs 2009, 69:1555-1623.

17. Vila J, Martinez JL: Clinical impact of the over-expression of efflux pump in nonfermentative Gram-negative bacilli, development of efflux pump inhibitors. Curr Drug Targets 2008, 9:797-807.

18. Hocquet D, Muller A, Blanc K, Plesiat P, Talon D, Monnet DL, Bertrand X: Relationship between antibiotic use and incidence of MexXY-OprM overproducers among clinical isolates of Pseudomonas aeruginosa. Antimicrob Agents Chemother 2008, 52:1173-1175.

19. Jeannot K, Sobel ML, El Garch F, Poole K, Plesiat P: Induction of the MexXY efflux pump in Pseudomonas aeruginosa is dependent on drugribosome interaction. J Bacteriol 2005, 187:5341-5346.

20. Clinical and Laboratory Standards Instittute: Methods for Dilution Antimicrobial Susceptibility Tests for Bacteria That Grow Aerobically: Seventeenth Edition M07-A7 Wayne, PA, USA, CLSI 2006.

21. Clinical and Laboratory Standards Instittute: Performace Standards for Antimicrobial Susceptibility Testing: Nineteenth Informational Supplement M100-S19 Wayne, PA, USA, CLSI 2009

22. Pfaller MA, Hollis RJ, Sader HS: Molecular biology - PFGE analysis of chromosomal restriction fragments. Clinical Microbiology Procedures Handbook Washington, DC: ASMlsenberg HD 1992, 10.5.

23. Toleman MA, Simm AM, Murphy TA, Gales AC, Biedenbach DJ, Jones RN, Walsh TR: Molecular characterization of SPM-1, a novel metallo-betalactamase isolated in Latin America: report from the SENTRY antimicrobial surveillance programme. J Antimicrob Chemother 2002, 50:673-679.

24. Mendes RE, Kiyota KA, Monteiro J, Castanheira M, Andrade SS, Gales AC, Pignatari AC, Tufik S: Rapid detection and identification of metallo-betalactamase-encoding genes by multiplex real-time PCR assay and melt curve analysis. J Clin Microbiol 2007, 45:544-547.

25. Picao RC, Poirel L, Gales AC, Nordmann P: Further identification of CTX-M2 extended-spectrum beta-lactamase in Pseudomonas aeruginosa. Antimicrob Agents Chemother 2009, 53:2225-2226.

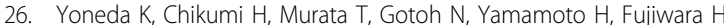
Nishino T, Shimizu E: Measurement of Pseudomonas aeruginosa multidrug efflux pumps by quantitative real-time polymerase chain reaction. FEMS Microbiol Lett 2005, 243:125-131.

27. El Amin N, Giske CG, Jalal S, Keijser B, Kronvall G, Wretlind B: Carbapenem resistance mechanisms in Pseudomonas aeruginosa: alterations of porin OprD and efflux proteins do not fully explain resistance patterns observed in clinical isolates. APMIS 2005, 113:187-196.

28. Savli H, Karadenizli A, Kolayli F, Gundes S, Ozbek U, Vahaboglu H: Expression stability of six housekeeping genes: A proposal for resistance 
gene quantification studies of Pseudomonas aeruginosa by real-time quantitative RT-PCR. J Med Microbiol 2003, 52:403-408.

29. Dumas $J$, van Delden C, Perron K, Kohler T: Analysis of antibiotic resistance gene expression in Pseudomonas aeruginosa by quantitative real-time-PCR. FEMS Microbiol Lett 2006, 254:217-225.

30. Muller PY, Janovjak H, Miserez AR, Dobbie Z: Processing of gene expression data generated by quantitative real-time RT-PCR. Biotechniques 2002, 32:1372-1379.

31. Hocquet D, Roussel-Delvallez M, Cavallo JD, Plesiat P: MexAB-OprM- and MexXY-overproducing mutants are very prevalent among clinical strains of Pseudomonas aeruginosa with reduced susceptibility to ticarcillin. Antimicrob Agents Chemother 2007, 51:1582-1583.

32. Rodriguez-Martinez JM, Poirel L, Nordmann P: Molecular epidemiology and mechanisms of carbapenem resistance in Pseudomonas aeruginosa. Antimicrob Agents Chemother 2009, 53:4783-4788.

doi:10.1186/1471-2180-10-217

Cite this article as: Xavier et al:: Efflux pumps expression and its association with porin down-regulation and $\beta$-lactamase production among Pseudomonas aeruginosa causing bloodstream infections in Brazil. BMC Microbiology 2010 10:217.

\section{Submit your next manuscript to BioMed Central} and take full advantage of:

- Convenient online submission

- Thorough peer review

- No space constraints or color figure charges

- Immediate publication on acceptance

- Inclusion in PubMed, CAS, Scopus and Google Scholar

- Research which is freely available for redistribution

Submit your manuscript at www.biomedcentral.com/submit 